\title{
Extracellular volume quantification in isolated hypertension - changes at the detectable limits?
}

Thomas A. Treibel ${ }^{1 \dagger}$, Filip Zemrak ${ }^{2 \dagger}$, Daniel M. Sado ${ }^{1}$, Sanjay M. Banypersad ${ }^{1}$, Steven K. White ${ }^{1,3}$, Viviana Maestrini ${ }^{1}$, Andrea Barison ${ }^{1,4}$, Vimal Patel ${ }^{1}$, Anna S. Herrey ${ }^{1}$, Ceri Davies ${ }^{2}$, Mark J. Caulfield ${ }^{2}$, Steffen E. Petersen ${ }^{2}$ and James C. Moon ${ }^{1,5^{*}}$

\begin{abstract}
Background: Diffuse myocardial fibrosis (DMF) is important in cardiovascular disease, however until recently could only be assessed by invasive biopsy. We hypothesised that DMF measured by T1 mapping is elevated in isolated systemic hypertension.

Methods: In a study of well-controlled hypertensive patients from a specialist tertiary centre, 46 hypertensive patients (median age 56, range 21 to 78, $52 \%$ male) and 50 healthy volunteers (median age 45, range 28 to 69, $52 \%$ male) underwent clinical CMR at $1.5 \mathrm{~T}$ with T1 mapping (ShMOLLI) using the equilibrium contrast technique for extracellular volume (ECV) quantification. Patients underwent 24-hours Automated Blood Pressure Monitoring (ABPM), echocardiographic assessment of diastolic function, aortic stiffness assessment and measurement of NT-pro-BNP and collagen biomarkers.

Results: Late gadolinium enhancement (LGE) revealed significant unexpected underlying pathology in 6 out of 46 patients ( $13 \%$; myocardial infarction $n=3$; hypertrophic cardiomyopathy $(H C M) n=3$ ); these were subsequently excluded. Limited, non-ischaemic LGE patterns were seen in 11 out of the remaining 40 (28\%) patients. Hypertensives on therapy (mean 2.2 agents) had a mean ABPM of 152/88 mmHg, but only $35 \%(14 / 40)$ had left ventricular hypertrophy ( $\left(\mathrm{VH} ; \mathrm{LV}\right.$ mass male $>90 \mathrm{~g} / \mathrm{m}^{2}$; female $>78 \mathrm{~g} / \mathrm{m}^{2}$ ). Native myocardial T1 was similar in hypertensives and controls ( $955 \pm 30 \mathrm{~ms}$ versus $965 \pm 38 \mathrm{~ms}, p=0.16$ ). The difference in ECV did not reach significance $(0.26 \pm 0.02$ versus $0.27 \pm 0.03, p=0.06)$. In the subset with $L V H$, the $E C V$ was significantly higher $(0.28 \pm 0.03$ versus $0.26 \pm 0.02, p<0.001)$.
\end{abstract}

Conclusion: In well-controlled hypertensive patients, conventional CMR discovered significant underlying diseases (chronic infarction, HCM) not detected by echocardiography previously or even during this study. T1 mapping revealed increased diffuse myocardial fibrosis, but the increases were small and only occurred with LVH.

Keywords: Hypertension, Left ventricular hypertrophy, Magnetic resonance imaging, T1 mapping, Myocardial fibrosis

\section{Background}

Arterial hypertension is one of the most common cardiovascular diseases and a major cause of morbidity and mortality in the developed world. Arterial hypertension results in increasing arterial stiffness and afterload, leading to remodelling of the myocardium due to

\footnotetext{
* Correspondence: J.Moon@ucl.ac.uk

${ }^{\dagger}$ Equal contributors

'Department of Cardiology, The Heart Hospital, University College London Hospitals NHS Trust, London, UK

${ }^{5}$ The Heart Hospital Imaging Centre, University College London Hospitals NHS Trust, 16-18 Westmoreland Street, London W1G 8PH, UK

Full list of author information is available at the end of the article
}

cardiomyocyte hypertrophy, fibroblast stimulation and then increased collagen formation. Progressive accumulation of interstitial collagen fibres, i.e. diffuse myocardial fibrosis (DMF), in left ventricular hypertrophy (LVH) has been shown at necropsy [1] and endomyocardial biopsy [2-4]. The cardiovascular magnetic resonance (CMR) derived late gadolinium enhancement (LGE) technique has shown patchy, non-specific or nonischaemic patterns of fibrosis in hypertension [5]. However, LGE is only able to detect relative increases between "normal" myocardium and focal scar and cannot therefore be used to identify and quantify absolute 
diffuse fibrosis [6-8]. This problem can be now addressed by quantification of $\mathrm{T} 1$ relaxation time mapping before and after gadolinium contrast administration and subsequent quantification of extracellular volume (ECV). Both ECV and native myocardial T1 have been shown to closely reflect the degree of histologic DMF [9-12]. Preliminary work on DMF in hypertension have described elevated extracellular volume fraction (ECV) compared to controls [13, 14]. However, this technique has not been used to comprehensively assess a cohort of hypertensive patients. We hypothesize that diffuse myocardial fibrosis measured by T1 mapping and ECV quantification is elevated in isolated systemic hypertension, correlates with cardiac remodelling and established biomarkers, and may therefore be a key biomarker in assessing the cardiac effects of systemic hypertension.

\section{Methods}

All research was carried out at University College London Hospital NHS Trust in collaboration with William Harvey Research Institute at Queen Mary University of London between April 2011 and February 2012. The study was approved by the ethical committee of UK National Research Ethics Service and conformed to the principles of the Helsinki Declaration (UK NRES 07/H0715/101). All subjects gave written consent to participate in the study.

Hypertensive subjects were recruited prospectively from a specialist hypertension clinic in a tertiary referral hospital. All patients had been investigated for secondary hypertension as part of their clinical work-up in the specialist hypertension clinic. Eligible patients were men and women between 18 and 80 years of age with essential hypertension. In accordance with the 2011 UK hypertension guidelines [15], ambulatory blood pressure measurement (ABPM) was used to confirm diagnosis of recruited patients (clinic blood pressure of $\geq 140$ / $90 \mathrm{mmHg}$ and daytime ABPM of $\geq 135 / 85 \mathrm{mmHg}$ ) and patients with "white coat" hypertension (not on antihypertensive medications with a normal ABPM) were excluded. Comprehensive assessment on the day of the CMR consisted of clinical history, arterial stiffness and blood pressure measurement following a period of rest, transthoracic echocardiography, electrocardiogram (ECG), blood tests (NT-pro-BNP, full blood count for the haematocrit, renal function, and lipid profile), 6minute walk test (6MWT), and CMR (including equilibrium diffuse myocardial fibrosis protocol). ECG was analysed for LVH by Cornell product and Sokolow-Lyon voltage criteria $[16,17]$.

A control group of healthy, normotensive volunteers recruited from hospital, university, community and general practice settings in Greater London, UK, were gender matched to the hypertension cohort. None were referred as patients for a clinical CMR scan that then turned out to be normal. All normal subjects had no history or symptoms of cardiovascular disease or diabetes. All subjects had a normal blood pressure (defined as $\leq 140 / 90 \mathrm{mmHg}$ ), 12-lead ECG and clinical CMR scan.

Exclusion criteria for both groups included diabetes mellitus, known ischaemic heart disease, contraindication to CMR (pacemakers) or gadolinium administration (glomerular filtration rate $<30 \mathrm{~mL} / \mathrm{min} / \mathrm{m}^{2}$ ). Healthy volunteers were excluded if they had a history of cardiovascular symptoms, an abnormal ECG or abnormal CMR.

\section{CMR protocol}

Standard CMR examinations were performed in all patients using a 1.5-T scanner (Avanto; Siemens Medical Imaging, Erlangen, Germany) in line with standard CMR protocols [18]. T1 mapping for CMR ECV quantification was performed using the Shortened Modified LookLocker Inversion recovery technique (ShMOLLI) (21) prior to contrast and at contrast equilibrium [9]. The studies were performed by cardiologists with $\geq 2$ years of experience in CMR imaging (DS, SB, SW). Standard LGE assessment using a fast low angle single shot inversion recovery sequence was used to assess focal myocardial fibrosis. Fifteen minutes after an initial contrast bolus of $0.1 \mathrm{mmol} / \mathrm{kg}$ of gadoterate meglumine (Dotarem; Guerbet, Paris, France) during which LGE images were acquired, an infusion of contrast at a rate of $0.0011 \mathrm{mmol} / \mathrm{kg}$ per minute was administered during which time the patient was removed from the scanner. After a minimum of 30 minutes the patient was returned into the scanner and equilibrium contrast $\mathrm{T} 1$ maps were acquired. The ECV was calculated by: $\mathrm{ECV}=(1$-haematocrit $) \times\left(1 / \Delta \mathrm{T} 1_{\text {tissue }}\right) /$ $\left(1 / \Delta \mathrm{T} 1_{\text {blood }}\right)$. Haematocrit was measured on the same day.

\section{CMR image analysis}

Left ventricular (LV) volumes, ejection fraction and mass were calculated using standard techniques [18] and analysed using a thresholding method indexed to body surface area. The presence of LGE was determined using the visual assessment of two authors (TT and JCM, the latter of whom has $>10$ years of CMR experience). For T1 measurements, a region of interest (ROI) was manually drawn on the septum on each image (TT) as shown in Fig. 1. Our group has previously validated this method (23). Myocardial feature tracking was performed by a blinded experienced observer using dedicated vector-based analysis tool (2D performance analysis MR, Tomtec, Unterschleissheim, Germany), as previously described [19]. Briefly, based on a contour manually drawn by an expert reader along the LV endocardial border of one frame, the software automatically propagates the contour and follows its features throughout the remainder of the cardiac cycle. LV short axis circumferential and radial strains were calculated from a mid-ventricular 


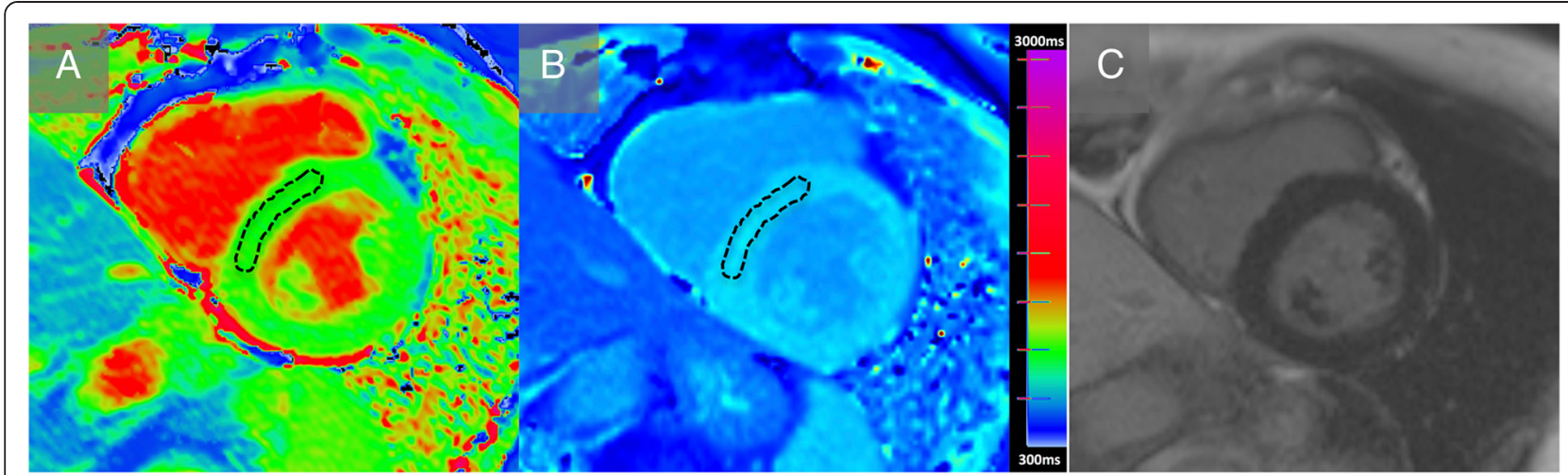

Fig. 1 Native and post contrast T1 mapping and LGE imaging in hypertension. Basal short axis T1 maps acquired pre-contrast (a) and post contrast (b) with ShMOLLI. Image c shows the corresponding FLASH LGE image. The dashed black line represents an example region of interest.

short-axis view containing both papillary muscles. Strain values (\% change from baseline) were obtained for each segment and global values defined as the mean of all segmental values.

\section{Echocardiography}

Echocardiography was performed on the day of the CMR in all hypertensive subjects using a GE Vivid E9 system (GE Healthcare, Wauwatosa, USA) with a 4-MHz transducer. Measurements were made according to European Society of Echocardiography criteria [20]. Early (E) and atrial (A) doppler mitral inflow wave velocities, pulmonary vein doppler, and tissue doppler of septal and lateral mitral annulus E' and A'-wave velocities were recorded. Diastolic function was graded according to the mitral inflow pattern, pulmonary vein in-flow and tissue doppler indices at the mitral valve annulus as previously described [21].

\section{Arterial stiffness}

Arterial stiffness was measured in hypertensive subjects using the Vicorder device (Skidmore Medical Ltd, UK), by registering carotid femoral pulse wave velocity (PWV), central aortic blood pressure and augmentation index as previously described and validated. $(23,24)$ PWV is the most validated method to non-invasively measure arterial stiffness. It is considered the gold standard assessment of aortic stiffness, as it is a relatively simple method with reported accuracy, reproducibility and is also an independent and strong predictor of adverse outcomes in a variety of common diseases, such as coronary heart disease and hypertension [22, 23]. The measurements were acquired in a separate quiet room after a period of rest. Central blood pressure measurement and aortic stiffness estimated by pulse wave velocity (PWV) measurement are more predictive of cardiovascular outcome than peripheral BP [23, 24].

\section{Biomarkers}

Serum levels of the N-terminal pro-hormone of probrain-natriuretic-peptide (NT-pro-BNP) were measured using a 2-site electrochemiluminescence immunoassay on a Roche E170 analyzer. Collagen Type I (procollagen type I carboxy-terminal propeptide, (PICP)) and Type III (Amino terminal peptide of type III procollagen ((PIIINP)) synthesis were measured using commercially available assays. The samples were collected at the time of CMR visit, immediately centrifuged and plasma samples were stored at $-80{ }^{\circ} \mathrm{C}$.

\section{Data analysis and statistics}

Statistical analysis was performed in $\mathrm{R}$ programming language for statistical computing (version 3.0.1, The $\mathrm{R}$ Foundation for Statistical Computing) and SPSS version 21 (SPSS Inc, Chicago, Ill). Normality of continuous data was assessed by visual inspection of the histograms and confirmed by the Shapiro-Wilk test. Continuous variables were expressed as mean $\pm \mathrm{SD}$ and non-parametric variables as median with inter-quartile range. Group mean comparisons were performed using Student's t-test (two groups) for normally distributed data or the MannWhittney $U$ test for skewed data. A probability value of $\mathrm{p}<0.05$ was considered statistically significant. Simple and multivariable linear regression models determined relationships between LV mass index, native myocardial $\mathrm{T} 1$ time, equilibrium myocardial $\mathrm{T} 1$ time and ECV as outcome variables, using demographic data, laboratory results and CMR measurements as exposure variables.

\section{Results}

Fifty-six well-controlled hypertensive patients were recruited. Ten patients were subsequently excluded as they were found to have white coat hypertension. Forty-six patients and 50 healthy volunteers underwent the full CMR protocol. Morphological, functional and LGE 
assessment revealed unexpected significant underlying pathology in 6 out of the 46 patients (13\%; chronic infarct $(\mathrm{n}=3)$; hypertrophic cardiomyopathy $(\mathrm{HCM}, \mathrm{n}=3)$; Fig. 2). These six patients were excluded from subsequent analysis. The remaining cohort comprised of 40 patients (median age 56, range 21 to $78,52 \%$ male) and 50 healthy volunteers (median age 45, range 28 to 69, $52 \%$ male). Patient ethnicity was self-reported as Caucasian/white in $72.5 \%$, Asian in $12.5 \%$ and AfroCaribbean/black in $15 \%$. Fifteen (37.5\%) of the study subjects were treated with angiotensin converting enzyme inhibitors, 11 (27.5\%) with angiotensin II antagonists, 25 (62.5 \%) with calcium channel blockers, 11 $(27.5 \%)$ with thiazide diuretic, $10(25 \%)$ with betablockers, $10(25 \%)$ with alpha-blockers $4(10 \%)$ with loop diuretic, $2(5 \%)$ with moxonidine and 1 (2.5\%) each with spironolactone, direct renin antagonist and phenoxybenzamine. Seven patients were not on any anti-hypertensive therapy. All clinical parameters are summarized in Table 1.

\section{Left ventricular remodelling}

The conventional CMR parameters are shown in Table 2. Hypertensive subjects had higher LV mass index
(83.9 \pm 33.6 vs. $\left.65.7 \pm 14.5 \mathrm{~g} / \mathrm{m}^{2}, \mathrm{p}<0.01\right)$, maximal wall thickness, LV mass to volume ratio (MVR), left atrial area (LAA) measured in the horizontal long axis view, indexed stroke volume (SV), but no significant differences in ejection fraction (EF) or indexed end-diastolic (EDV) and end-systolic (ESV) volumes.

$\mathrm{LVH}$ (defined as LV mass index $>90 \mathrm{~g} / \mathrm{m}^{2}$ in males and $>78 \mathrm{~g} / \mathrm{m}^{2}$ in females [25]) was found in $35 \%$ of patients (14/40). Those who fulfilled LVH criteria had higher maximal wall thickness, but also larger ventricles (as expressed by ESV and EDV) and larger indexed LAA (Table 3). Furthermore, this subgroup had higher central blood pressure, higher QRS complex voltage on ECG (by Sokolov and Cornell) and more advanced diastolic dysfunction $(\mathrm{p}<0.01)$ (Table 3$)$.

Higher LV mass was associated with larger LV volumes and LA size (Table 4): $10.3 \mathrm{~g}$ for each $10 \mathrm{ml}$ increase in $\operatorname{ESV}(p<0.01), 7.4 \mathrm{~g}$ for each $10 \mathrm{ml}$ increase in EDV $(p<0.0001)$ and $5.4 \mathrm{~g}$ for each $1 \mathrm{~cm}^{2}$ increase in LAA $(p<0.0001)$. The severity of diastolic dysfunction (trans-mitral E/A ratio, tissue Doppler lateral E/E' ratio) and higher ECG voltage parameters (by Cornell and Sokolov) also increased with higher LV mass (data not presented). There were no differences between the groups

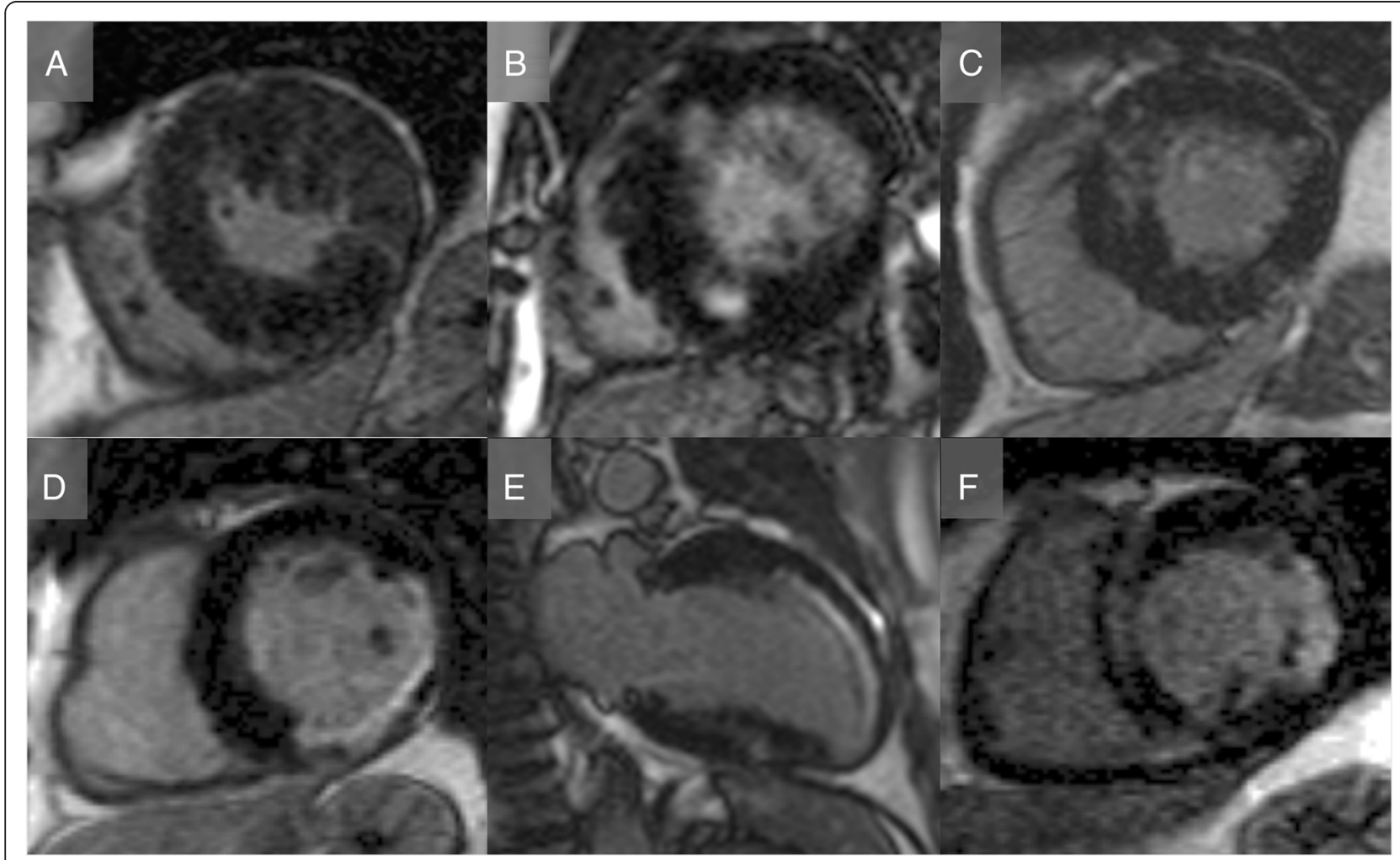

Fig. 2 Diagnosis of occult disease by LGE in patients with arterial hypertension. Standard late gadolinium enhancement assessment using a fast low angle single shot inversion recovery sequence revealed six patients (13\% of cohort) with either hypertrophic cardiomyopathy (a-c) or infarct pattern (d-f). 
Table 1 Baseline clinical characteristics for healthy volunteers and hypertensives

\begin{tabular}{|c|c|c|c|}
\hline & $\begin{array}{l}\text { Healthy volunteers } \\
(\mathrm{n}=50)\end{array}$ & $\begin{array}{l}\text { Hypertensive } \\
(n=40)\end{array}$ & $p$ value \\
\hline Men & $26(52 \%)$ & $21(53 \%)$ & 0.96 \\
\hline Age in years (IQR) & 44 (32.0 to 54.8$)$ & 58.5 (49.0 to 65.5$)$ & $<0.001$ \\
\hline \multicolumn{4}{|l|}{ Ethnicity } \\
\hline Whites & $41(82 \%)$ & $28(70 \%)$ & 0.12 \\
\hline Blacks & $8(16 \%)$ & $6(15 \%)$ & 0.59 \\
\hline Asians & $1(2 \%)$ & $6(15 \%)$ & 0.06 \\
\hline Height (cm) & $171.9 \pm 11.3$ & $170.2 \pm 9.1$ & 0.41 \\
\hline Weight (kg) & $75.9 \pm 13.8$ & $86.6 \pm 15.7$ & $<0.01$ \\
\hline Body surface area (m2) & $1.9 \pm 0.2$ & $2.0 \pm 0.2$ & $<0.05$ \\
\hline Body mass index (kg/m2) & $25.6 \pm 3.2$ & $29.8 \pm 4.5$ & $<0.0001$ \\
\hline Systolic blood pressure (mmHg) & $122.7 \pm 10.5$ & $152.0 \pm 17.2$ & $<0.0001$ \\
\hline Diastolic blood pressure (mmHg) & $74.1 \pm 9.1$ & $88.1 \pm 10.7$ & $<0.0001$ \\
\hline eGFR (ml/min/1.73 m2) & $91.2 \pm 16.5$ & $81.1 \pm 20.8$ & $<0.05$ \\
\hline Haematocrit (\%) & $0.42 \pm 0.03$ & $0.42 \pm 0.04$ & 0.37 \\
\hline Number of antihypertensives (0/1/2/3/4/5/6) & & $7 / 5 / 13 / 7 / 4 / 3 / 1$ & \\
\hline
\end{tabular}

in the systolic strain parameters derived from feature tracking analysis, in particular in global longitudinal, circumferential or radial strain.

\section{Late gadolinium enhancement}

On LGE imaging, and after exclusion of the six patients with infarct and HCM LGE patterns (Fig. 2), 11 of the remaining 40 hypertensive subjects $(28 \%)$ had nonspecific LGE either at the right ventricular insertion point, papillary muscle or patchy mid-wall enhancement (Fig. 3). These LGE patterns were seen more often with increasing degrees of $\mathrm{LVH}$, evidence of cardiac remodelling (higher LV mass index, mass volume ratio, wall thickness) and increased afterload (higher systolic and diastolic pressure, and also augmentation index), but not with age, sex or ethnicity. Healthy controls did not have LGE.

\section{T1 mapping and ECV quantification}

Native myocardial T1, equilibrium-contrast T1 and ECV values are shown in Table 5 . Native myocardial T1 was similar in patients and controls $(955 \pm 30 \mathrm{~ms}$ versus $965 \pm$ $38 \mathrm{~ms} ; p=0.16$ ), however native myocardial T1 times were longer in patients with LVH than in ones without LVH $(997 \pm 27 \mathrm{~ms}$ vs. $948 \pm 31 \mathrm{~ms} ; p<0.001)$. Equilibriumcontrast myocardial $\mathrm{T} 1$ was shorter in patients than controls $(578 \pm 37 \mathrm{~ms}$ vs. $618 \pm 33 \mathrm{~ms} ; p<0.0001)$. The difference was attributable to higher weight $(86.6 \pm 15.7 \mathrm{~kg}$ vs. $75.9 \pm 13.8 \mathrm{~kg}, p<0.01$ ), which resulted in higher absolute total contrast dose, and to worse eGFR $(81 \pm 21 \mathrm{ml} /$ $\mathrm{min} / \mathrm{m}^{2}$ vs. $91 \pm 17 \mathrm{ml} / \mathrm{min} / \mathrm{m}^{2}, \mathrm{p}<0.05$ ), which also shortened equilibrium-contrast blood T1 (478 $\pm 56 \mathrm{~ms}$ vs. $517 \pm 48 \mathrm{~ms} ; p<0.001)$.

The difference in ECV between the hypertensives and controls did not reach significance $(27.1 \pm 2.7 \%$ vs.

Table 2 Cardiovascular Magnetic Resonance Parameters

\begin{tabular}{llll}
\hline & $\begin{array}{l}\text { Healthy volunteers } \\
(\mathrm{n}=50)\end{array}$ & $\begin{array}{l}\text { Hypertensive } \\
(\mathrm{n}=40)\end{array}$ & $p$ value \\
\hline EDV index $(\mathrm{ml} / \mathrm{m} 2)$ & $73.2 \pm 13.6$ & $71.2 \pm 17.0$ & 0.7 \\
ESV index $(\mathrm{ml} / \mathrm{m} 2)$ & $25.0($ IQR: 21.0 to 29.75$)$ & $21.8($ IQR: 15.3 to 28.1$)$ & 0.13 \\
LVEF $(\%)$ & $66.5 \pm 5.8$ & $69.0 \pm 8.9$ & $11.7 \pm 2.5$ \\
LAA index $(\mathrm{cm} / \mathrm{m} 2)$ & $11.1 \pm 1.8$ & $11.9 \pm 2.4$ & 0.12 \\
Maximal wall thickness $(\mathrm{mm})$ & $6.7 \pm 1.2$ & $76.9($ IQR: 66.5 to 88.4$)$ & $<.16$ \\
LV mass index $(\mathrm{g} / \mathrm{m} 2)$ & $65.0($ IQR: 55.3 to 74.5$)$ & $1.09(\mathrm{IQR}: 0.93$ to 1.31$)$ & $14(35 \%)$
\end{tabular}


Table 3 Hypertensives without versus Hypertensives with LVH

\begin{tabular}{|c|c|c|c|}
\hline & $\begin{array}{l}\text { Hypertensives no LVH } \\
\mathrm{n}=26\end{array}$ & $\begin{array}{l}\text { Hypertensives with LVH } \\
\mathrm{n}=14\end{array}$ & $p$-value \\
\hline \multicolumn{4}{|l|}{ T1 mapping } \\
\hline T1 Blood (ms) & $1564 \pm 61$ & $1614 \pm 83$ & 0.02 \\
\hline T1 Myocardium (ms) & $948 \pm 31$ & $997 \pm 27$ & $<0.001$ \\
\hline ECV by ShMOLLI (\%) & $26.2 \pm 2.2$ & $28.8 \pm 2.8$ & $<0.01$ \\
\hline \multicolumn{4}{|l|}{ Clinical } \\
\hline Men & $16(62 \%)$ & $5(35 \%)$ & 0.12 \\
\hline Age in years (IQR) & $57.8 \pm 12.2$ & $53.2 \pm 17.5$ & 0.4 \\
\hline Height (cm) & $171 \pm 10.4$ & $168.5 \pm 6.1$ & 0.44 \\
\hline Weight (kg) & $86.6 \pm 16.9$ & $86.6 \pm 13.8$ & 1 \\
\hline Body surface area $\left(\mathrm{m}^{2}\right)$ & $2.02 \pm 0.24$ & $2.01 \pm 0.18$ & 0.79 \\
\hline Body mass index $\left(\mathrm{kg} / \mathrm{m}^{2}\right)$ & $29.5 \pm 4.7$ & $30.4 \pm 4.2$ & 0.49 \\
\hline Systolic blood pressure $(\mathrm{mmHg})$ & $150.1 \pm 18.0$ & $155.5 \pm 15.7$ & 0.35 \\
\hline Diastolic blood pressure $(\mathrm{mmHg})$ & $87.1 \pm 11.7$ & $90.0 \pm 8.4$ & 0.35 \\
\hline eGFR $\left(\mathrm{ml} / \mathrm{min} / 1.73 \mathrm{~m}^{2}\right)$ & $83.6 \pm 18.8$ & $76.4 \pm 24.2$ & 0.23 \\
\hline Haematocrit (\%) & $0.43 \pm 0.03$ & $0.42 \pm 0.05$ & 0.39 \\
\hline Number of antihypertensives $(0 / 1 / 2 / 3 / 4 / 5 / 6)$ & $3 / 5 / 8 / 6 / 2 / 1 / 1$ & $4 / 0 / 5 / 1 / 2 / 2 / 0$ & \\
\hline \multicolumn{4}{|l|}{ CMR } \\
\hline EDV index $\left(\mathrm{ml} / \mathrm{m}^{2}\right)$ & $66.2 \pm 11.7$ & $82.5 \pm 20.5$ & 0.02 \\
\hline ESV index $\left(\mathrm{ml} / \mathrm{m}^{2}\right)$ & $19.9 \pm 7.2$ & $29.4 \pm 14.4$ & 0.05 \\
\hline LVEF (\%) & $70.7 \pm 7.3$ & $65.9 \pm 10.9$ & 0.16 \\
\hline LAA index $\left(\mathrm{cm} / \mathrm{m}^{2}\right)$ & $10.9 \pm 2.1$ & $13.3 \pm 2.5$ & $<0.01$ \\
\hline Maximal wall thickness (mm) & $10.9 \pm 1.6$ & $13.8 \pm 2.6$ & $<0.001$ \\
\hline LV mass index $\left(\mathrm{g} / \mathrm{m}^{2}\right)$ & $68.6 \pm 11.8$ & $112.4 \pm 39.6$ & $<0.001$ \\
\hline LV mss/EDV (g/ml) & $1.07 \pm 0.26$ & $1.41 \pm 0.5$ & 0.05 \\
\hline Transverse strain & $34.0 \pm 11.8$ & $30.8 \pm 9.4$ & 0.21 \\
\hline Longitudinal strain & $-18.7 \pm 5.4$ & $-17.4 \pm 3.8$ & 0.41 \\
\hline Circumferential strain & $-24.7 \pm 5.3$ & $-24.6 \pm 3.8$ & 0.64 \\
\hline Radial strain & $37.3 \pm 13.1$ & $35.4 \pm 7.1$ & 0.94 \\
\hline Late enhancement, n (\%) & $5(19)$ & $6(43)$ & 0.15 \\
\hline \multicolumn{4}{|l|}{ Electrocardiogram } \\
\hline Sokolov-Lyon index & $22.3 \pm 4.8$ & $32.5 \pm 13.1$ & $<0.01$ \\
\hline Cornell voltage criteria & $13.7 \pm 5.2$ & $21.1 \pm 8.7$ & $<0.01$ \\
\hline \multicolumn{4}{|l|}{ Aortic stiffness } \\
\hline Pulse wave velocity & $7.7 \pm 1.5$ & $8.4 \pm 1.6$ & 0.15 \\
\hline Augmentation index & $17.0 \pm 5.7$ & $13.6 \pm 6.6$ & 0.18 \\
\hline Aortic systolic blood pressure (mmHg) & $141.5 \pm 14.9$ & $167.4 \pm 27.2$ & $<0.01$ \\
\hline Aortic pulse pressure $(\mathrm{mmHg})$ & $54.6 \pm 13.4$ & $64.3 \pm 17.2$ & 0.13 \\
\hline \multicolumn{4}{|l|}{ Diastolic function on echocardiogram } \\
\hline$E / A$ & $0.9 \pm 0.3$ & $1.1 \pm 0.5$ & 0.7 \\
\hline E/e' septal & $10.3 \pm 2.9$ & $12.9 \pm 7.3$ & 0.36 \\
\hline E/e' lateral & $8.0 \pm 2.3$ & $12.98 \pm 6.4$ & $<0.01$ \\
\hline Diastolic grade (I/II/III/IV) & $3 / 18 / 5 / 0$ & $0 / 6 / 7 / 1$ & $<0.01$ \\
\hline
\end{tabular}


Table 4 Univariate Predictors of T1 mapping Parameters and Left Ventricular Hypertrophy

\begin{tabular}{|c|c|c|c|c|c|c|c|c|}
\hline & \multicolumn{2}{|c|}{ Native myocardial T1 } & \multicolumn{2}{|c|}{ Equilibrium myocardial T1 } & \multirow{2}{*}{\multicolumn{2}{|c|}{$\begin{array}{l}\text { ECV } \\
\text { Univariable }\end{array}$}} & \multirow{2}{*}{\multicolumn{2}{|c|}{$\begin{array}{l}\text { LV mass index } \\
\text { Univariable }\end{array}$}} \\
\hline & \multicolumn{2}{|c|}{ Univariable } & \multicolumn{2}{|c|}{ Univariable } & & & & \\
\hline & beta & $p$ & beta & $p$ & beta & $p$ & beta & $p$ \\
\hline Men & -9.2 & 0.2 & 2.49 & 0.77 & -1.27 & $<0.05$ & 18 & $<0.001$ \\
\hline Age per year & -0.22 & 0.32 & -0.09 & $<0.001$ & 0 & 0.18 & -0.15 & 0.39 \\
\hline Ethnicity & 9.45 & 0.11 & & & & & & \\
\hline Asian & & & -3.5 & 0.76 & 0.46 & 0.5 & 0.97 & 0.89 \\
\hline Black/Afrocaribbean & & & -37.5 & $<0.05$ & 4.07 & $<0.0001$ & 37.45 & $<0.001$ \\
\hline Height (cm) & -0.13 & 0.72 & 0.61 & 0.14 & -0.05 & $<0.05$ & 0.46 & 0.08 \\
\hline Weight (kg) & 0.11 & 0.64 & -0.82 & $<0.01$ & -0.03 & 0.14 & 0.51 & $<0.01$ \\
\hline Body surface area $\left(\mathrm{m}^{2}\right)$ & 4.13 & 0.8 & -39.5 & $<0.05$ & -2.0 & 0.09 & 35.0 & $<0.01$ \\
\hline Body mass index $\left(\mathrm{kg} / \mathrm{m}^{2}\right)$ & 0.74 & 0.37 & -4.75 & $<0.0001$ & -0.02 & 0.72 & 1.48 & $<0.05$ \\
\hline Systolic blood pressure (mmHg) & 0.13 & 0.5 & -0.76 & $<0.001$ & 0.01 & 0.33 & 0.37 & $<0.05$ \\
\hline Diastolic blood pressure (mmHg) & 0.07 & 0.81 & -1.15 & $<0.01$ & 0.01 & 0.62 & 0.60 & $<0.05$ \\
\hline eGFR $\left(\mathrm{ml} / \mathrm{min} / 1.73 \mathrm{~m}^{2}\right)$ & 0.09 & 0.66 & 0.88 & $<0.0001$ & -0.03 & 0.09 & -0.42 & $<0.01$ \\
\hline Hematocrit (\%) & -0.94 & 0.42 & 0.38 & 0.8 & -0.23 & $<0.01$ & 179.7 & $<0.05$ \\
\hline EDV index $\left(\mathrm{ml} / \mathrm{m}^{2}\right)$ & 0.35 & 0.15 & 0.08 & 0.77 & 0.03 & 0.9 & 0.74 & $<0.0001$ \\
\hline ESV index $\left(\mathrm{ml} / \mathrm{m}^{2}\right)$ & 0.23 & 0.55 & 0.13 & 0.77 & 0.01 & 0.67 & 1.03 & $<0.001$ \\
\hline LVEF (\%) & 0.09 & 0.85 & -0.18 & 0.76 & 0.04 & 0.32 & -0.68 & 0.06 \\
\hline LAA index $\left(\mathrm{cm} / \mathrm{m}^{2}\right)$ & 3.12 & 0.06 & -0.59 & 0.77 & 0.34 & $<0.01$ & 5.14 & $<0.0001$ \\
\hline Maximal wall thickness (mm) & 2.89 & $<0.01$ & -5.02 & $<0.0001$ & 0.18 & $<0.05$ & - & - \\
\hline LV mass index $\left(\mathrm{g} / \mathrm{m}^{2}\right)$ & 0.40 & $<0.01$ & -0.33 & $<0.05$ & 0.02 & $<0.05$ & - & - \\
\hline LVH (presence) & 44.26 & $<0.0001$ & -28.8 & $<0.05$ & 2.65 & $<0.001$ & - & - \\
\hline E/A ratio & -0.15 & 0.99 & 16.2 & 0.3 & -0.001 & 0.9 & 40.2 & $<0.01$ \\
\hline E/e' septum & 1.81 & 0.14 & -1.51 & 0.2 & 0.002 & 0.08 & 3.18 & $<0.01$ \\
\hline E/e' lateral wall & 3.21 & $<0.01$ & -1.23 & 0.3 & 0.001 & 0.3 & 3.76 & $<0.001$ \\
\hline
\end{tabular}

$26.1 \pm 2.4 \%, p=0.06$ ). Hypertensive patients with LVH had higher ECV than the ones without LVH $(28.8 \pm 2.8 \%$ vs. $26.2 \pm 2.2 \%, p<0.01$ ).

The relationship between T1 mapping and clinical parameters are summarized in Table 4:

Longer native myocardial T1 was associated with the presence of LVH $(\beta=44.3, p<0.0001)$ and $\mathrm{LVH}$ measures: higher maximal wall thickness $(\beta=2.89, p<$ $0.01)$ and LV mass index $(\beta=0.4, p<0.01)$. Native myocardial T1 was also prolonged with higher E/e' ratio in the lateral wall $(\beta=3.2, p<0.01)$, higher central aortic pressure $(\beta=0.7, p<0.01)$ and higher ECG voltage sum by Cornell $(\beta=1.13, p=0.01)$. The associations with indexed left atrial area $(\beta=3.12, p=0.06)$, E/A ratio $(\beta=-0.15, p=0.99)$ and $\mathrm{E} / \mathrm{e}$ ' septum $(\beta=1.81, p=0.14)$ were not significant.

Equilibrium-contrast myocardial T1 was shorter in older subjects $(\beta=-0.09$ per year, $p<0.001)$, in AfroCaribbean/blacks $(\beta=-37.5, \quad p<0.05)$ and subjects with $\operatorname{LVH}(\beta=2.65, p<0.001)$. Equilibrium-contrast myocardial T1 shortened by $5 \mathrm{~ms}$ for each millimetre increase in maximal wall thickness $(p<0.001)$, by $7.6 \mathrm{~ms}$ for $10 \mathrm{mmHg}$ higher systolic blood pressure $(p<0.001)$ and by $11.5 \mathrm{~ms}$ for each $10 \mathrm{mmHg}$ higher diastolic blood pressure $(p<0.01)$. It was also shorter with worsening eGFR ( $\beta=0.88, p<0.0001)$ and strongly associated with equilibrium-contrast blood T1 time $(\beta=0.66, p<0.0001)$, however after adjustment, only the equilibrium-contrast blood and myocardium T1 times remained their association $(\beta=0.6, p<0.0001)$. There was neither an association between equilibrium-contrast myocardial $\mathrm{T} 1$ and left atrial area $(\beta=-0.59, p=0.77)$ nor parameters of diastolic dysfunction.

ECV was higher in women $(\beta=1.27, p<0.05)$ and Afro-Caribbean/blacks $(\beta=4.1, p<0.0001)$. Taller subjects had lower ECV $(\beta=-0.05$ per $\mathrm{cm}, p<0.05)$. One $\%$ increase in haematocrit was associated with $0.23 \%$ reduction in ECV $(p<0.01)$. Similarly to equilibrium-contrast myocardial T1, ECV increased with LV hypertrophy: 


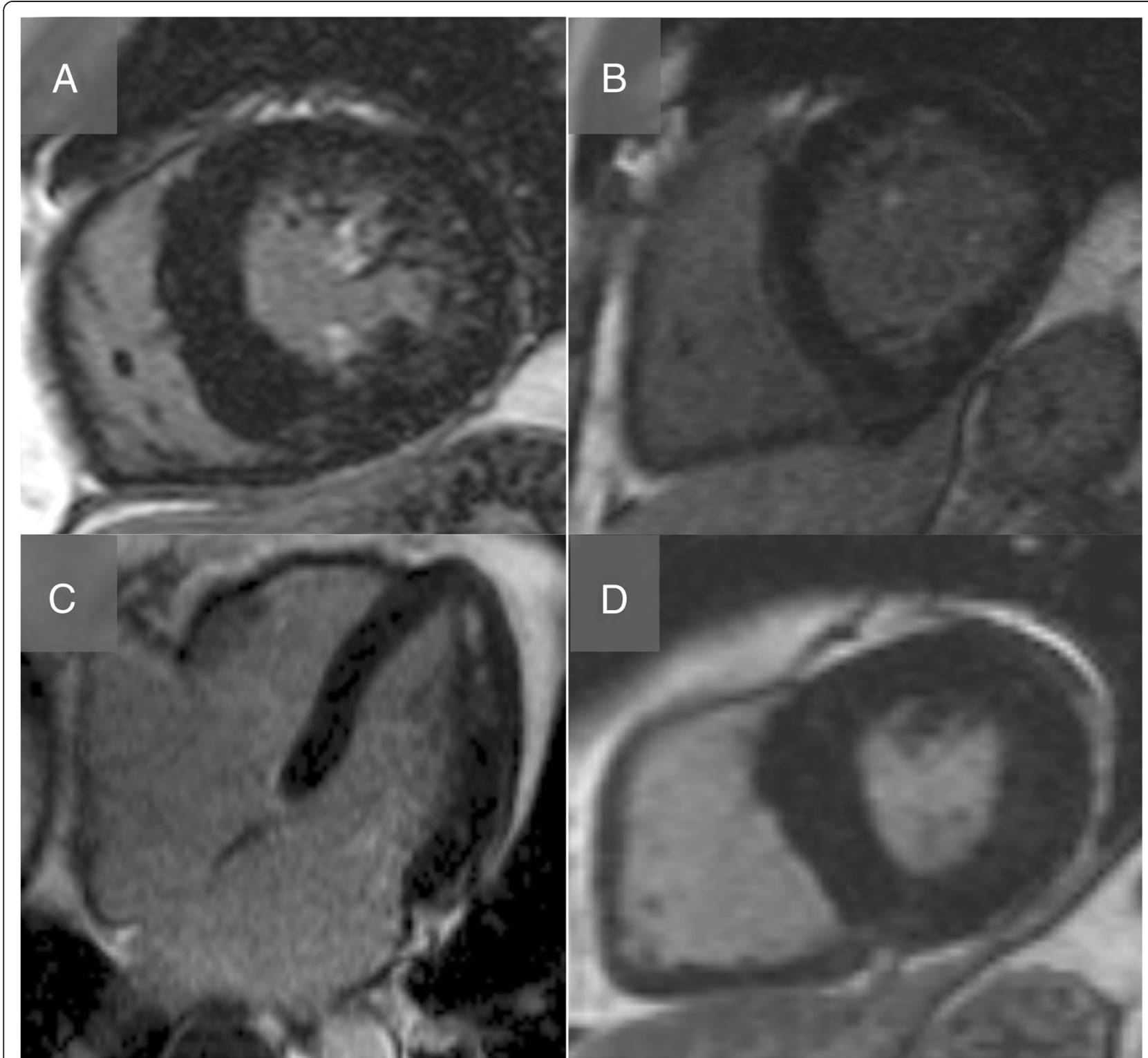

Fig. 3 Non-ischaemic, non-HCM pattern of late gadolinium enhancement. Standard late gadolinium enhancement assessment using a fast low angle single shot inversion recovery sequence revealed non-ischaemic, non-HCM pattern of focal fibrosis in $13 \%$ of the hypertensive cohort, with LGE in the papillary muscles $(A+B)$ mid-wall $(B+C)$ and right ventricular insertion points $(\mathbf{d})$.

Table 5 T1 Mapping Parameters

\begin{tabular}{llll}
\hline & $\begin{array}{l}\text { Healthy Volunteers } \\
(\mathrm{n}=50)\end{array}$ & $\begin{array}{l}\text { Hypertensive } \\
(\mathrm{n}=40)\end{array}$ & p value \\
\hline $\begin{array}{l}\text { Pre-contrast/Native } \\
\text { T1 Blood (ms) }\end{array}$ & $1568 \pm 71$ & $1581 \pm 72$ & 0.4 \\
T1 Myocardium (ms) & $955 \pm 30$ & $965 \pm 38$ & 0.16 \\
Post-contrast at & & & \\
Equilibrium & & $478 \pm 56$ & $<0.001$ \\
T1 Blood (ms) & $517 \pm 48$ & $578 \pm 37$ & $<0.001$ \\
T1 Myocardium (ms) & $618 \pm 33$ & $27.1 \pm 2.7$ & 0.06 \\
ECV by ShMOLLI (\%) & $26.1 \pm 2.4$ & & \\
\hline
\end{tabular}

maximal wall thickness $(\beta=0.18$ per $\mathrm{mm}, p<0.05)$, LV mass index $(\beta=0.02, p<0.05)$ and with Cornell's index $(\beta=0.12, p<0.05)$. ECV also increased by $0.34 \%$ for each $1 \mathrm{~cm} / \mathrm{m}^{2}$ increase in LAA (but there was no significant association with parameters of diastolic dysfunction). ECV was independent of eGFR and blood pressure values. After adjustment only Afro-Caribbean race $(\beta=2.94$, $p<0.01)$, height $(\beta=-0.06, p<0.05)$ and native T1 time $(\beta=0.04, p<0.0001)$ were associated with ECV.

\section{Functional status and biomarkers}

ECV also predicted functional status was predicted by $\mathrm{ECV}$, with a one per cent in ECV shortening the distance 
on the 6-MWT by 14 meters $(p<0.05)$. Native and equilibrium T1 times were not associated with worsening functional status.

NT-pro-BNP was only available for the hypertensive cohort and ranged between 4 and 100 (median 8 pmol/ L). NT-pro-BNP was higher in hypertensives with $\mathrm{LVH}$ than without $\mathrm{LVH}(26.9 \pm 16.4$ vs $12.0 \pm 19.6$ $\mathrm{pmol} / \mathrm{L}, p<0.001)$. Log-transformed NT-pro-BNP correlated very weakly with markers of DMF (native myocardial T1, $\beta=0.005, p<0.01$; equilibrium-contrast T1 $\beta=-0.004, p<0.05$; ECV $\beta=0.05, p<0.05$ ).

Collagen biomarkers were available for ten healthy volunteers and 31 patients. PICP $(43.0 \pm 3.9$ vs $54.3 \pm$ $2.3 \mathrm{ug} / \mathrm{L}, p=0.02)$ and PIIINP (3.7 \pm 0.1 vs $4.5 \pm 0.2 \mathrm{ug} / \mathrm{L}$, $p<0.01$ ) were higher in patients than in the healthy controls. There was no significant difference between $\mathrm{LVH}+$ ve and LVH-ve hypertensive patients. There was no association between collagen biomarker levels and myocardial T1 mapping parameters.

\section{Discussion}

This study has shown that DMF assessed by T1 mapping increases in hypertensive patients, but that the changes were small, and occurred only in those with $\mathrm{LVH}$, in keeping with prior findings using other modalities [26].

This study was of well-controlled isolated hypertension. Conventional CMR with LGE frequently adds value by identifying other pathologies - here that value was unexpectedly high $-13 \%$ of patients had new diagnoses of hypertrophic cardiomyopathy or silent infarction. Although echocardiography is the first imaging modality for the assessment of LVH due to greater availability and smaller cost, CMR is the gold standard for LV volume and function assessment and has the advantage of allowing tissue characterisation with LGE and T1 mapping.

After exclusion of these other pathologies, 11 of the remaining 40 hypertensive subjects (28\%) had nonspecific patchy LGE either at the right ventricular insertion point, in the papillary muscles or LV mid-wall enhancement. Although there is no data on the predictive value of non-ischaemic LGE in isolated arterial hypertension, it tracked increasing degree of LV hypertrophy and LV afterload. Furthermore, nonischaemic LGE in the prototypical LV afterload disease of aortic stenosis is predictive of mortality [27].

Hypertensive patients had lower equilibrium-contrast myocardial T1 and a trend to higher ECV. Native myocardial T1 and ECV values (but not post-contrast T1) were higher in hypertensives with than without LVH. Native T1 is a composite signal of the intra- and extracellular myocardial compartments, whereas after administration of contrast (equilibrium-contrast T1 and ECV) the signal from the extra-cellular space dominates. The lower equilibrium-contrast $\mathrm{T} 1$ values in hypertensives were attributable to increased weight (increased contrast bolus) and worse renal function. The results suggest that hypertensive patients have increased myocardial fibrosis (both focal and diffuse), but that really this is triggered with the onset of LVH rather than earlier in the pathogenesis. There was some signal from those without LVH but rather than being an early sign, this could be residual from fibrosis prior to LVH regression. In contrast to recent findings by Kuruvilla et al. [28], who found reduced peak systolic circumferential strain in LVH + ve hypertensive patients compared to $\mathrm{LVH}$-ve and controls, we did not find a significant difference, which may be due aggressive anti-hypertensive treatment with associated anti-fibrotic effects ( $70 \%$ of $\mathrm{LVH}+$ ve patients were on an ACE-inhibitor/angiotensin II or aldosterone receptor antagonist).

Ultimately, the size of the study, severity of the disease and level of control of hypertension (65\% of our subjects had no LVH) rendered this study underpowered to further elucidate all the questions; specifically, there was a lack of significant and consistent association across parameters of T1 mapping and diastolic dysfunction as well as strain. Diffuse fibrosis appears not to be a major early player in treated hypertensive heart disease prior to the onset of $\mathrm{LVH}$. A larger prospective study would be necessary to scrutinise changes prior to and post therapy.

The T1 mapping field is rapidly advancing. The signal is very large in amyloid, increased in iron load, Anderson-Fabry disease, myocarditis and focal fibrosis (e.g. infarction), but smaller in diffuse fibrosis. This study suggests current technology is hitting limits for subtle changes, such as might be seen in well-controlled hypertension without LVH. Several positive studies have been published [29-31], but highlighting technological limits is as important and this effectively negative study has taken longer to get submitted than mainly positive studies, a common bias in new research fields.

\section{Study limitations and perspective}

Patients were recruited from a specialist hypertension clinic and therefore had very well-controlled disease with documented good adherence to medication. A significant number of patients were on anti-hypertensive medications, which are attributed to have anti-fibrotic effects, which may have resulted in a reduction of $\mathrm{LVH}$ and ECV compared to untreated subjects, however the sample size was too small to explore this. We used good technology - ShMOLLI T1 mapping with equilibrium imaging. But the use of the equilibrium infusion technique, which requires re-positioning of the patient and does not allow co-registration of pre and post contrast maps, is not necessary for this disease. We 
now know the equilibrium is un-necessary for low ECV increases [32, 33]: recent work by our group and others has shown that the bolus-only technique has good agreement with histological fibrosis, with a small degree of ECV overestimation in the high ECV range [32-34]. Whether the latest techniques with new adiabatic pulses, sampling schemes, motion correction, new reconstructions (e.g. T1* for blood), ECV mapping and new methodologies to minimise partial voluming errors will make a difference is unknown currently [35].

\section{Conclusion}

In well-controlled hypertensive patients, conventional CMR discovered significant underlying diseases (chronic infarction and HCM). T1 mapping based assessment suggested small increases in DMF, occurring only in those patients with LVH. This study highlights that interstitial changes in early hypertension (pre-LVH) are small and not detectable by current iterations of $\mathrm{T} 1$ mapping technique.

\section{ENDNOTE - Biomarker description}

Carboxy-terminal propeptide of procollagen type I (PICP): Levels were measured in EDTA plasma by sandwich enzyme linked immunosorbent assay (ELISA) KIT manufactured by USCN Life Science Inc, Democratic Republic of China). The minimal detectable level of $26.6 \mathrm{pg} / \mathrm{mL}$.

Amino-terminal peptide of type III procollagen (PIIINP): Levels were measured in EDTA plasma using a competitive radioimmunoassay (RIA) assay manufactured by Orion Diagnostica, Finland. The minimal detectable level was $0.3 \mathrm{ug} / \mathrm{L}$.

\begin{abstract}
Abbreviations
ABPM: Ambulatory blood pressure measurement; CMR: Cardiovascular magnetic resonance; DMF: Diffuse myocardial fibrosis; ECG: Electrocardiogram; ECV: Extracellular volume; EDV: End-diastolic volume; EF: Ejection fraction; ESV: End-systolic volume; HCM: Hypertrophic cardiomyopathy; LGE: Late gadolinium enhancement; LAA: Left atrial area; LV: Left ventricle; LVH: Left ventricular hypertrophy; MVR: Mass to volume ratio; NHS: National Health Service; PICP: Procollagen type I carboxy-terminal propeptide; PIIINP: Procollagen type III n-terminal propeptide; PWV: Pulse wave velocity; ROI: Region of interest; ShMOLLI: Shortened modified look-locker inversion recovery technique; SV: Stroke volume; 6MWT: 6 minutes walk test.

\section{Competing interests}

The authors declare that they have no competing interests and no relationships relevant to the contents of this paper to disclose.

\section{Authors' contributions \\ $\Pi T$ and FZ coordinated and performed all steps of this study. DS, SB, SW participated in the CMR acquisition. VM analysed all ECG data. VP coordinated the analysis of the biomarkers. $\mathrm{AH}$ and $\mathrm{CD}$ participated in the coordination and review of the CMR data. T, FZ, MC, SEP, JM conceived of the study, and participated in its design and coordination and helped to} draft the manuscript. All authors read and approved the final manuscript.
\end{abstract}

Authors' information

Thomas A Treibel and Filip Zemrak are Joint first authors.

\section{Acknowledgement}

\section{Sources of funding}

The funding source (British Heart Foundation and UK National Institute for Health Research) provided salaries for research training (FZ, TT, DS, SW), but had no role in study design, collection, analysis, interpretation, writing, or decisions with regard to publication. This work was undertaken at University College London Hospital, which received a proportion of funding from the UK Department of Health National Institute for Health Research Biomedical Research Centres funding scheme. We are grateful to King's College London Laboratories for processing the collagen biomarker panel.

\section{Author details}

'Department of Cardiology, The Heart Hospital, University College London Hospitals NHS Trust, London, UK. ${ }^{2}$ National Institute for Health Research Cardiovascular Biomedical Research Unit at Barts, William Harvey Research Institute, Barts and the London School of Medicine and Dentistry, Queen Mary University of London, London, UK. ${ }^{3}$ The Hatter Cardiovascular Institute, University College London Hospitals NHS Trust, London, UK. ${ }^{4}$ Fondazione Toscana Gabriele Monasterio and Scuola Superiore Sant'Anna, Pisa, Italy. ${ }^{5}$ The Heart Hospital Imaging Centre, University College London Hospitals NHS Trust, 16-18 Westmoreland Street, London W1G 8PH, UK.

Received: 18 May 2015 Accepted: 21 July 2015

Published online: 12 August 2015

\section{References}

1. Rossi MA. Pathologic fibrosis and connective tissue matrix in left ventricular hypertrophy due to chronic arterial hypertension in humans. J Hypertens. 1998:16:1031-41

2. Pardo Mindan FJ, Panizo A. Alterations in the extracellular matrix of the myocardium in essential hypertension. Eur Heart J. 1993;14:12-14.

3. Schwartzkopff B, Motz W, Frenzel H, Vogt M, Knauer S, Strauer BE. Structural and functional alterations of the intramyocardial coronary arterioles in patients with arterial hypertension. Circulation. 1993:88:993-1003.

4. Mundhenke M, Schwartzkopff B, Strauer BE. Structural analysis of arteriolar and myocardial remodelling in the subendocardial region of patients with hypertensive heart disease and hypertrophic cardiomyopathy. Virchows Arch. 1997:431:265-73.

5. Rudolph A, Abdel-Aty H, Bohl S, Boye P, Zagrosek A, Dietz R, et al. Noninvasive detection of fibrosis applying contrast-enhanced cardiac magnetic resonance in different forms of left ventricular hypertrophy relation to remodeling. J Am Coll Cardiol. 2009;53:284-91.

6. Zemrak F, Petersen SE. Late gadolinium enhancement CMR predicts adverse cardiovascular outcomes and mortality in patients with coronary artery disease: systematic review and meta-analysis. Prog Cardiovasc Dis. 2011;54:215-29.

7. Ismail TF, Prasad SK, Pennell DJ. Prognostic importance of late gadolinium enhancement cardiovascular magnetic resonance in cardiomyopathy. Heart. 2012;98:438-42.

8. Otto CM, Burwash IG, Legget ME, Munt BI, Fujioka M, Healy NL, et al. Prospective study of asymptomatic valvular aortic stenosis. Clinical, echocardiographic, and exercise predictors of outcome. Circulation. 1997;95:2262-70

9. Flett AS, Hayward MP, Ashworth MT, Hansen MS, Taylor AM, Elliott PM, et al. Equilibrium contrast cardiovascular magnetic resonance for the measurement of diffuse myocardial fibrosis: preliminary validation in humans. Circulation. 2010;122:138-44.

10. Kehr E, Sono M, Chugh SS, Jerosch-Herold M. Gadolinium-enhanced magnetic resonance imaging for detection and quantification of fibrosis in human myocardium in vitro. Int J Cardiovasc Imaging. 2008;24:61-8.

11. Miller CA, Naish JH, Bishop P, Coutts G, Clark D, Zhao S, et al. Comprehensive validation of cardiovascular magnetic resonance techniques for the assessment of myocardial extracellular volume. Circ Cardiovasc Imaging. 2013;6:373-83.

12. Bull S, White SK, Piechnik SK, Flett AS, Ferreira VM, Loudon M, et al. Human non-contrast $\mathrm{T} 1$ values and correlation with histology in diffuse fibrosis. Heart. 2013:99(13):932-7.

13. Janardhanan R, Adenaw N, Jiji R, Brooks J, Epstein FH, Kramer CM, et al. Quantifying myocardial fibrosis in hypertensive left ventricular hypertrophy using T1 mapping. J Cardiovasc Magn Reson. 2012;14:1-2. 
14. Mongeon F-P, Jerosch-Herold M, Coelho-Filho OR, Seabra LF, Watanabe E, Blankstein $\mathrm{R}$, et al. Identification of myocardial extracellular matrix expansion by cardiac MRI in hypertensive patients. J Cardiovasc Magn Reson. 2011;13:0109.

15. National Clinical Guideline Centre (UK). Hypertension: The Clinical Management of Primary Hypertension in Adults: Update of Clinical Guidelines 18 and 34. London: National Institute for Health and Clinical Excellence: Guidance]; 2011

16. Sokolow M, Lyon TP. The ventricular complex in left ventricular hypertrophy as obtained by unipolar precordial and limb leads. Am Heart J. 1949;37:161-86.

17. Okin PM, Roman MJ, Devereux RB, Kligfield P. Electrocardiographic identification of increased left ventricular mass by simple voltage-duration products. J Am Coll Cardiol. 1995;25:417-23.

18. Kramer CM, Barkhausen J, Flamm SD, Kim RJ, Nagel E. Standardized cardiovascular magnetic resonance imaging (CMR) protocols, society for cardiovascular magnetic resonance: board of trustees task force on standardized protocols. J Cardiovasc Magn Reson. 2008;10:35.

19. Hor KN, Gottliebson WM, Carson C, Wash E, Cnota J, Fleck R, et al. Comparison of magnetic resonance feature tracking for strain calculation with harmonic phase imaging analysis. JACC Cardiovasc Imaging. 2010;3:144-51.

20. Evangelista A, Flachskampf F, Lancellotti P, Badano L, Aguilar R, Monaghan $M$, et al. European Association of Echocardiography recommendations for standardization of performance, digital storage and reporting of echocardiographic studies. Eur J Echocardiogr. 2008;9:438-48.

21. Osranek M, Seward JB, Buschenreithner B, Bergler-Klein J, Heger M, Klaar U, et al. Diastolic function assessment in clinical practice: the value of 2-dimensional echocardiography. Am Heart J. 2007;154:130-6.

22. Asmar R, Benetos A, Topouchian J, Laurent P, Pannier B, Brisac AM, et al. Assessment of arterial distensibility by automatic pulse wave velocity measurement. Validation and clinical application studies. Hypertension. 1995;26:485-90.

23. Lehmann ED, Parker JR, Hopkins KD, Taylor MG, Gosling RG. Validation and reproducibility of pressure-corrected aortic distensibility measurements using pulse-wave-velocity Doppler ultrasound. J Biomed Eng. 1993;15:221-8.

24. Mattace-Raso FU, van der Cammen TJ, Hofman A, van Popele NM, Bos ML, Schalekamp MA, et al. Arterial stiffness and risk of coronary heart disease and stroke: the Rotterdam Study. Circulation. 2006;113:657-63.

25. Maceira AM, Prasad SK, Khan M, Pennell DJ. Normalized left ventricular systolic and diastolic function by steady state free precession cardiovascular magnetic resonance. J Cardiovasc Magn Reson. 2006;8:417-26.

26. Muller-Brunotte R, Kahan T, Lopez B, Edner M, Gonzalez A, Diez J, et al. Myocardial fibrosis and diastolic dysfunction in patients with hypertension: results from the Swedish Irbesartan Left Ventricular Hypertrophy Investigation versus Atenolol (SILVHIA). J Hypertens. 2007;25:1958-66.

27. Dweck MR, Joshi S, Murigu T, Alpendurada F, Jabbour A, Melina G, et al. Midwall fibrosis is an independent predictor of mortality in patients with aortic stenosis. J Am Coll Cardiol. 2011;58:1271-9.

28. Kuruvilla S, Janardhanan R, Antkowiak P, Keeley EC, Adenaw N, Brooks J, et al. Increased extracellular volume and altered mechanics are associated with LVH in hypertensive heart disease, not hypertension alone. JACC Cardiovasc Imaging. 2015:8:172-80.

29. Sado DM, Flett AS, Banypersad SM, White SK, Maestrini V, Quarta G, et al. Cardiovascular magnetic resonance measurement of myocardial extracellular volume in health and disease. Heart. 2012;98:1436-41.

30. Liu CY, Chang Liu Y, Wu C, Armstrong A, Volpe GJ, van der Geest RJ, et al. Evaluation of Age related Interstitial Myocardial Fibrosis with Cardiac Magnetic Resonance Contrast-Enhanced T Mapping in the Multi-ethnic Study of Atherosclerosis (MESA). J Am Coll Cardiol. 2013;62(14):1280-7.

31. Maestrini V, Treibel TA, White SK, Fontana M, Moon JC. T1 Mapping for Characterization of Intracellular and Extracellular Myocardial Diseases in Heart Failure. Curr Cardiovasc Imaging Rep. 2014;7:9287.

32. White SK, Sado DM, Fontana M, Banypersad SM, Maestrini V, Flett AS, et al. T1 mapping for myocardial extracellular volume measurement by CMR: bolus only versus primed infusion technique. JACC Cardiovasc Imaging. 2013;6:955-62.

33. Schelbert EB, Testa SM, Meier CG, Ceyrolles WJ, Levenson JE, Blair AJ, et al. Myocardial extravascular extracellular volume fraction measurement by gadolinium cardiovascular magnetic resonance in humans: slow infusion versus bolus. J Cardiovasc Magn Reson. 2011;13:16.

34. Kawel N, Nacif M, Zavodni A, Jones J, Liu S, Sibley CT, et al. T1 mapping of the myocardium: intra-individual assessment of post-contrast $\mathrm{T} 1$ time evolution and extracellular volume fraction at 3T for Gd-DTPA and Gd-BOPTA. J Cardiovasc Magn Reson. 2012;14:26.

35. Moon JC, Messroghli DR, Kellman P, Piechnik SK, Robson MD, Ugander M, et al. Myocardial T1 mapping and extracellular volume quantification: a Society for Cardiovascular Magnetic Resonance (SCMR) and CMR Working Group of the European Society of Cardiology consensus statement. J Cardiovasc Magn Reson. 2013;15:92.

\section{Submit your next manuscript to BioMed Central and take full advantage of:}

- Convenient online submission

- Thorough peer review

- No space constraints or color figure charges

- Immediate publication on acceptance

- Inclusion in PubMed, CAS, Scopus and Google Scholar

- Research which is freely available for redistribution 